\title{
TWO THEOREMS CONCERNING FUNCTIONS HOLOMORPHIC ON MULTIPLY CONNECTED DOMAINS
}

BY E. L. STOUT ${ }^{1}$

Communicated by Walter Rudin, March 25, 1963

1. Let $\Omega$ be a finitely connected plane domain whose boundary, $\partial \Omega$, consists of the circles $\Gamma_{0}, \Gamma_{1}, \cdots, \Gamma_{n}$. We assume $\Gamma_{j}$ lies in the interior of $\Gamma_{0}$ for $j=1,2, \cdots, n$. Let $\Delta_{0}$ be the interior of $\Gamma_{0}$ and let $\Delta_{j}$ be the exterior of $\Gamma_{j}, j=1,2, \cdots, n$. We then have $\Omega=\bigcap_{j=0}^{n} \Delta_{j}$. Let $H_{\infty}[\Omega]$ be the collection of all bounded holomorphic functions in $\Omega$. We shall say that a set $S$ of points of $\Omega$ is an interpolation set for $\Omega$ if given a bounded complex valued function $w$ on $S$ there is $f \in H_{\infty}[\Omega]$ such that $f(z)=w(z)$ for all $z \in S$. If $\left\{z_{n}\right\}_{n=1}^{\infty}$ is a sequence in $\Omega$, without limit points in $\Omega$, we write $\left\{z_{n}\right\}=S_{0} \cup S_{1} \cup \ldots \cup S_{n}$ where the $S_{j}$ are pairwise disjoint and where the only limit points of $S_{j}$ lie in $\Gamma_{j}$, $j=0,1, \cdots, n$.

In the present note we sketch proofs for the following two theorems:

THEOREM A. The sequence $\left\{z_{n}\right\}$ is an interpolation set for $\Omega$ if and only if each $S_{j}$ is an interpolation set for the disc $\Delta_{j}$.

Theorem B. Let $f_{1}, f_{2}, \cdots, f_{m}$ be functions in $H_{\infty}[\Omega]$ such that $\left|f_{1}(z)\right|+\left|f_{2}(z)\right|+\cdots+\left|f_{m}(z)\right| \geqq \delta>0$ for all $z \in \Omega$. Then there exist functions $g_{1}, g_{2}, \cdots, g_{m} \in H_{\infty}[\Omega]$ such that $f_{1} g_{1}+f_{2} g_{2}+\cdots+f_{m} g_{m}=1$.

L. Carleson [2] has established Theorem B in case $\Omega$ is the open unit disc. He has also proved [1] that the sequence $\left\{z_{n}\right\}_{n=1}^{\infty}$ is an interpolation sequence for the open unit disc if and only if there is a $\delta>0$ such that

$$
\prod_{n \neq k}\left|\frac{z_{n}-z_{k}}{1-\bar{z}_{n} z_{k}}\right|>\delta
$$

for $k=1,2,3, \cdots$. For a discussion and alternative proof see [3, pp. 194-208].

2. Outline of the proof of Theorem A. Let $B_{j}$ be the Blaschke product associated with the disc $\Delta_{j}$ and the set of points $S_{j}$, $j=0, \cdots, n$. Note that there is an $\eta>0$ such that $\left|B_{j}(z)\right|>\eta$ for $z \in S_{k}$ if $k \neq j$.

${ }^{1}$ National Science Foundation Graduate Fellow. 
Suppose now that $S_{j}$ is an interpolation set for $\Delta_{j}, j=0, \cdots, n$. Let $w$ be a bounded function on $S$, and let $f_{j} \in H_{\infty}\left[\Delta_{j}\right]$ be such that

$$
f_{j}(z)=w(z) /\left(B_{0}(z) \cdots B_{j-1}(z) B_{j+1}(z) \cdots B_{n}(z)\right)
$$

for all $z \in S_{j}$. Define

$$
F=f_{0} B_{1} B_{2} \cdots B_{n}+f_{1} B_{0} B_{2} \cdots B_{n}+\cdots+f_{n} B_{0} B_{1} \cdots B_{n-1} .
$$

Then $F \in H_{\infty}[\Omega]$ and $F(z)=w(z)$ for all $z \in S$.

Conversely, assume that $\left\{z_{n}\right\}_{n=1}^{\infty}$ is an interpolation set for $\Omega$. If $f \in H_{\infty}[\Omega]$ we define $\|f\|$ by

$$
\|f\|=\sup \{|f(z)|: z \in \Omega\} .
$$

A Banach space argument like that in [3, p. 196] shows that there is a constant $M$ such that if $w$ is a function on $\left\{z_{n}\right\}_{n=1}^{\infty}$ with $|w(z)| \leqq 1$ for all $z \in\left\{z_{n}\right\}_{n=1}^{\infty}$, then there is $f \in H_{\infty}[\Omega]$ with $\|f\| \leqq M$ and $f(z)=w(z)$, $z \in\left\{z_{n}\right\}_{n=1}^{\infty}$. Given $z_{k} \in S_{j}$, let $B_{j}^{(k)}$ be the Blaschke product associated with the disc $\Delta_{j}$ and the set $S_{j} \backslash\left\{z_{k}\right\}$. Let $f \in H_{\infty}[\Omega]$ be such that $f\left(z_{n}\right)=0$ for $n \neq k, f\left(z_{k}\right)=1$ and such that $\|f\| \leqq M$. The function

$$
g=f /\left(B_{0} \cdots B_{j-1} B_{j}^{(k)} B_{j+1} \cdots B_{n}\right)
$$

is in $H_{\infty}[\Omega]$. Since there is $\delta>0$ such that $\left|B_{i}(z)\right| \geqq \delta$ for all $z \in \Gamma_{j}$, $i \neq j$, we have that $\|g\| \leqq M / \delta^{n}$. In particular then $\left|g\left(z_{k}\right)\right| \leqq M / \delta^{n}$. This yields

$$
\left|\delta^{n} M^{-1} f\left(z_{k}\right) /\left(B_{0}\left(z_{k}\right) \cdots B_{j-1}\left(z_{k}\right) B_{j+1}\left(z_{k}\right) \cdots B_{n}\left(z_{k}\right)\right)\right| \leqq\left|B_{j}^{(k)}\left(z_{k}\right)\right| \text {. }
$$

Since $f\left(z_{k}\right)=1$, and the product $B_{0} \cdots B_{j-1} B_{j+1} \cdots B_{n}$ is uniformly bounded away from zero on $S_{j}$, we have that $B_{j}^{(k)}\left(z_{k}\right) \geqq \delta_{1}>0$. This estimate is uniform in $k$, so $S_{j}$ is an interpolation set for $\Delta_{j}$.

3. Outline of the proof of Theorem B. Observe that $H_{\infty}[\Omega]$ is a commutative Banach algebra with identity if it is given the norm defined by (1). Let $\mathfrak{M}[\Omega]$ be the maximal ideal space of $H_{\infty}[\Omega]$; we regard $\mathfrak{M}[\Omega]$ as the collection of all nonzero complex homomorphisms of $H_{\infty}[\Omega]$ with the weak* topology. Let $\mathfrak{M}_{e}[\Omega]$ be the collection of those homomorphisms $\phi_{\lambda}$ of the form $\phi_{\lambda}(f)=f(\lambda), \lambda \in \Omega$. It is known $\left[3\right.$, p. 163] that to establish our result it suffices to prove $\mathfrak{M}_{e}[\Omega]$ dense in $\mathfrak{M}[\Omega]$.

For $j=1,2, \cdots, n$, let $H_{\infty}^{0}\left[\Delta_{j}\right]$ be the closed subalgebra of $H_{\infty}[\Omega]$ consisting of those $f$ which are restrictions to $\Omega$ of functions in $H_{\infty}\left[\Delta_{j}\right]$ which vanish at infinity. It is known $[4$, p. 56] that if $f \in H_{\infty}[\Omega]$, then $f$ can be written in the form 


$$
f=f_{0}+f_{1}+\cdots+f_{n},
$$

$f_{0} \in H_{\infty}\left[\Delta_{0}\right], f_{j} \in H_{\infty}^{0}\left[\Delta_{j}\right], 1 \leqq j$. It is immediate that this decomposition is unique; it yields

$$
H_{\infty}[\Omega]=H_{\infty}\left[\Delta_{0}\right] \oplus H_{\infty}^{0}\left[\Delta_{1}\right] \oplus \cdots \oplus H_{\infty}^{0}\left[\Delta_{n}\right],
$$

the direct sum being understood in the sense of Banach spaces.

Following some ideas of I. J. Schark (see [3, p. 159, ff.]), we note that the function $z$ is in $H_{\infty}[\Omega]$. It gives rise to the function $z$ on $\mathfrak{M}[\Omega]$ given by $\hat{z}(\phi)=\phi(z)$. We can prove that $\hat{z}$ maps $\mathfrak{M}[\Omega]$ onto $\bar{\Omega}$ and that $z$ is one-to-one over $\Omega$. If $\alpha \in \partial \Omega$, set $\mathfrak{M}_{\alpha}=\{\phi \in \mathfrak{M}[\Omega]$ : $\hat{z}(\phi)=\alpha\}$. A slight modification of the argument for the disc case shows that if $f \in H_{\infty}[\Omega]$, then $\hat{f}$ is constant on $\mathfrak{M}_{\alpha}$ if and only if $f$ is continuously extensible to $\Omega \cup\{\alpha\}$ and that if $f$ is so extensible, then $\hat{f}(\phi)=f(\alpha)$ for all $\phi \in \mathfrak{M}[\Omega]$.

Suppose now that $\phi$ is a multiplicative linear functional defined on $H_{\infty}\left[\Delta_{0}\right]$ viewed as a subalgebra of $H_{\infty}[\Omega]$ by the direct sum decomposition (3). Let $\phi(z) \in \Omega$. Then $\phi$ admits a unique extension to an element of $\mathfrak{M}[\Omega]$. This is clear since $z$ maps $\mathfrak{M}[\Omega]$ onto $\bar{\Omega}$ and is oneto-one over $\Omega$. If $\alpha=\phi(z)$ lies in $\Gamma_{0}, \phi$ also admits a unique extension to an element of $\mathfrak{M}[\Omega]$. For uniqueness, suppose that $\phi^{*}$ is an extension of $\phi$ to all of $H_{\infty}[\Omega]$. For $f \in H_{\infty}[\Omega]$, write $f=f_{0}+f_{1}+\cdots+f_{n}$ in accordance with (2). The linearity of $\phi^{*}$ implies that $\phi^{*}(f)=\phi^{*}\left(f_{0}\right)$ $+\phi^{*}\left(f_{1}\right)+\cdots+\phi^{*}\left(f_{n}\right)$. Since $\phi^{*}$ is an extension of $\phi$, and since, for $j=1,2, \cdots, n, f_{j}$ is continuously extensible to $\Omega \cup\{\alpha\}$, it follows that $\phi^{*}(f)=\phi\left(f_{0}\right)+f_{1}(\alpha)+\cdots+f_{n}(\alpha)$. This establishes the uniqueness of the extension. This choice of $\phi^{*}$ yields a multiplicative functional. To see this, suppose $g \in H_{\infty}[\Omega]$ and write $g=g_{0}+g_{1}+\cdots+g_{n}$ by (2). Then $f g=\sum_{j, k=0}^{n} f_{j} g_{k}$. Since $\phi^{*}$ is plainly linear, we need only show $\phi^{*}\left(f_{j} g_{k}\right)=\phi^{*}\left(f_{j}\right) \phi^{*}\left(g_{k}\right)$. If neither $j$ nor $k$ is zero, $f_{j} g_{k}$ is continuously extensible to $\Omega \cup\{\alpha\}$, so we need only consider terms of the form $f_{0} g_{k}$ and $f_{j} g_{0}$. Suppose then that $f \in H_{\infty}\left[\Delta_{0}\right], g \in H_{\infty}\left[\Delta_{j}\right], j \neq 0$. Since $\phi^{*}(g)=g(\alpha)$, we are finished if we can show $\phi^{*}(f g-g(\alpha) f)=0$. Write

$$
f g-g(\alpha) f=h_{0}+h_{1}+\cdots+h_{n}
$$

in accordance with (2). Then $h_{j}$ is continuous at $\alpha$ for $j=1, \cdots, n$, and since $f g-g(\alpha) f$ is continuous at $\alpha$, it follows that $h_{0}$ must be continuous at $\alpha$ so that $\phi\left(h_{0}\right)=h_{0}(\alpha)$. Therefore $\phi^{*}(f g-g(\alpha) f)=h_{0}(\alpha)$ $+h_{1}(\alpha)+\cdots+h_{n}(\alpha)=0$. We conclude that $\phi^{*}$ is multiplicative.

If $\phi$ is a multiplicative linear functional on $H_{\infty}\left[\Delta_{0}\right]$ such that $\phi(z) \in \Gamma_{j}$ for $j \neq 0$, our argument indicates that $\phi$ admits many exten- 
sions to an element of $\mathfrak{M}[\Omega]$. If $\phi(z) \in \Delta_{0} \backslash \bar{\Omega}$, then $\phi$ admits no extension.

The same argument applies to $\Delta_{1}, \cdots, \Delta_{n}$ in place of $\Delta_{0}$. This also shows that every element of $\mathfrak{M}[\Omega]$ is determined by its action on the subalgebras $H_{\infty}\left[\Delta_{0}\right], H_{\infty}^{0}\left[\Delta_{1}\right], \cdots, H_{\infty}^{0}\left[\Delta_{n}\right]$. It now follows that $\mathfrak{M}_{e}[\Omega]$ is dense in $\mathfrak{M}[\Omega]$. For suppose $\phi \in \mathfrak{M}[\Omega]$, and suppose $\alpha=\phi(z)$ $\in \Gamma_{k}$. Let $\phi^{(j)}$ be the restriction of $\phi$ to the subalgebra $H_{\infty}\left[\Delta_{j}\right]$. By Carleson's result for the disc, there is a point $\lambda \in \Delta_{k}$ such that the point evaluation $\phi_{\lambda}^{(k)}$ at $\lambda$ is near $\phi^{(k)}$ in the sense of the weak* topology in the maximal ideal space of $H_{\infty}\left[\Delta_{k}\right]$. If $\lambda$ is near $\alpha$, then $\lambda \in \Omega$, and each of the point evaluations at $\lambda, \phi_{\lambda}^{(j)}$, for $j \neq k$ is near the point evaluation $\phi_{\alpha}^{(j)}$ in the maximal ideal space of $H_{\infty}\left[\Delta_{j}\right]$. But then the point evaluation $\phi_{\lambda} \in \mathfrak{M}_{e}[\Omega]$ is near the homomorphism $\phi$ in $\mathfrak{M}[\Omega]$. Thus $\mathfrak{M}_{e}[\Omega]$ is dense in $\mathfrak{M}[\Omega]$, and we have our result.

4. We can relax our condition on the boundary of $\Omega$ as follows. Our results are plainly invariant under conformal mapping. It is known $[5$, p. 377] that every finitely connected domain with no nondegenerate boundary components is conformally equivalent to a domain bounded by circles. Thus our results apply to this more general class of domains.

\section{REFERENCES}

1. L. Carleson, An interpolation problem for bounded analytic functions, Amer. J. Math. 80 (1958), 921-930.

2. - Interpolation by bounded analytic functions and the corona problem, Ann. of Math. (2) 76 (1962), 547-559.

3. K. Hoffman, Banach spaces of analytic functions, Prentice-Hall, Englewood Cliffs, N. J., 1962.

4. W. Rudin, Analytic functions of class $H_{p}$, Trans. Amer. Math. Soc. 78 (1955), $46-66$.

5. A. Hurwitz and R. Courant, Funktionentheorie, Springer, Berlin, 1922.

UNIVERSITY OF WISCONSIN 